\title{
Robust topological edge states and superconductivity in few-layer stanene
}

\section{Chenxiao Zhao}

Shanghai Jiao Tong University

\section{Leiqiang Li}

University of Science and Technology of China

\section{Liying Zhang}

University of Science and Technology of China

Jin Qin

Shanghai Jiao Tong University

\section{Haiyang Ma}

Shanghai Jiao Tong University

\section{Bing Xia}

Shanghai Jiao Tong University

\section{Bo Yang}

Shanghai Jiao Tong University

\section{Hao Zheng}

Shanghai Jiao Tong University https://orcid.org/0000-0002-6495-874X

\section{Shiyong Wang}

Shanghai Jiao Tong University https://orcid.org/0000-0001-6603-9926

Canhua Liu

Shanghai Jiao Tong University

\section{Yaoyi Li}

Shanghai Jiao Tong University

\section{Dandan Guan}

Shanghai Jiao Tong University https://orcid.org/0000-0002-3714-8813

\section{Ping Cui}

University of Science and Technology of China https://orcid.org/0000-0003-3196-2563

\section{Zhenyu Zhang}

University of Science and Technology of China https://orcid.org/0000-0001-5844-3558 Jinfeng Jia ( $\boldsymbol{\sigma}$ jjia@sjtu.edu.cn )

Shanghai Jiao Tong University https://orcid.org/0000-0002-9900-281X 
Keywords: stanene, topological superconductors, insulators

Posted Date: February 4th, 2021

DOI: https://doi.org/10.21203/rs.3.rs-173918/v1

License: (c) (1) This work is licensed under a Creative Commons Attribution 4.0 International License. Read Full License 


\title{
Robust topological edge states and superconductivity in few-layer stanene
}

\author{
Chenxiao Zhao ${ }^{1}$, Leiqiang $\mathrm{Li}^{2}$, Liying Zhang ${ }^{3,4}$, Jin Qin ${ }^{1}$, Haiyang $\mathrm{Ma}^{1}$, \\ Bing Xia ${ }^{1}$, Bo Yang ${ }^{1}$, Hao Zheng ${ }^{1,5,6}$, Shiyong Wang ${ }^{1,5,6}$, Canhua liu ${ }^{1,5,6}$, \\ Yaoyi Li ${ }^{1,5,6}$, Dandan Guan ${ }^{1}$, Ping Cui ${ }^{2 a}$, Zhenyu Zhang ${ }^{2}$, Jinfeng Jia ${ }^{1,5,6 b}$ \\ ${ }^{1}$ Key Laboratory of Artificial Structures and Quantum Control (Ministry of Education), \\ Shenyang National Laboratory for Materials Science, School of Physics and Astronomy, \\ Shanghai Jiao Tong University, Shanghai 200240, China \\ ${ }^{2}$ International Center for Quantum Design of Functional Materials (ICQD), \\ Hefei National Laboratory for Physical Sciences at Microscale (HFNL), \\ and CAS Center for Excellence in Quantum Information and Quantum Physics, \\ University of Science and Technology of China, Hefei, Anhui 230026, China. \\ ${ }^{3}$ Key Laboratory for Special Functional Materials of Ministry of Education, \\ Collaborative Innovation Center of Nano Functional Materials and Applications, \\ School of Materials Science and Engineering, \\ Henan University, Kaifeng, 475004, China. \\ ${ }^{4}$ International Laboratory for Quantum Functional Materials of Henan, \\ and School of Physics and Microelectronics, \\ Zhengzhou University, Zhengzhou 450001, China. \\ ${ }^{5}$ Tsung-Dao Lee Institute, Shanghai Jiao Tong University, Shanghai 200240, China \\ ${ }^{6}$ CAS Center for Excellence in Topological Quantum Computation, \\ University of Chinese Academy of Sciences, Beijing 100190, China
}

\footnotetext{
a cuipg@ustc.edu.cn

b jfjia@sjtu.edu.cn
} 


\begin{abstract}
Stanene was proposed to be a large-gap quantum spin Hall insulator, yet to date, convincing evidence of topological edge states in stanene remains to be seen, partly due to the fact that the topological property depends on the interplay between substrate, chemical functionalization, and layer thickness. Here we fabricate 1-5 layer high-quality stanene films on the $\mathrm{Bi}(111)$ substrate by using hydrogen atoms as surfactants, and demonstrate their strikingly robust nontrivial topology using scanning tunneling microscopy/spectroscopy and first-principles calculations. The observed topological edge states possess a bilateral-penetration depth shorter than $4 \mathrm{~nm}$, allowing the formation of dense and parallel multi-edge-channels. Our calculations further show that surface hydrogenation helps to improve the quality of stanene films, while the Bi substrate endows the films with robust nontrivial topology. These stanene films also exhibit superconductivity, and the coexistence of nontrivial topology and superconductivity renders them distinct potential to become the simplest one-dimensional topological superconductors.
\end{abstract}




\section{Introduction}

Quantum spin Hall insulators (QSHIs) are novel two-dimensional (2D) materials with insulating bulk interior and conducting edge channels that prohibit backscattering $[1,2]$. Among the massive candidates, stanene, the tin analogue of graphene, stands out due to its simple structure and large gap[3]. Theoretical studies have shown that stanene films may possess tunable topological properties that are sensitive to the substrate, chemical functionalization, and layer thickness[3-9]. Such sensitivities endow stanene with great tunability, but meanwhile pose challenges in experimental measurements. In previous studies, monolayer stanene films with compressed strain were shown to exhibit a trivial band structure[5, 10-12]. A major advance in realizing nontrivial band topology was achieved when growing stanene on the $\mathrm{Cu}(111)-(3 \times 3)$ surface, where the ultra-flat stanene films which are stable at low temperature show an inverted band order[13]. More recently, stanene films grown on the $\operatorname{InSb}(111)-(3 \times 3)$ surface were also shown to display the existence of edge states[14], even though the films are highly defective, probably caused by the large lattice mismatch between the overlayers and substrate. Identification of a proper substrate to grow stable stanene films with nontrivial topological properties is critically needed for further exploration and potential utilization of their salient topological properties. In particular, a clear and robust evidence of the one-dimensional (1D) topological edge states as characterized by the well-localized distribution of density of states (DOS) at edges remains to be seen. Such highly desirable advances, coupled with the recently confirmed superconductivity in the few-layer regime[15, 16], may also lead to potential realization of topological superconductivity[17] in such systems.

Here we have successfully grown 1-5 layer stanene on the $\operatorname{Bi}(111)$ substrate, where the stanene films are stable at room temperature. Our in-situ scanning tunneling microscopy/spectroscopy (STM/STS) measurements confirm the quality of the stanene films and show clear evidences of topological edge states in all the 1-5 layer films, which escapes the layer-dependence constraints of QSHIs proposed in previous studies[18, 19]. Meanwhile, a very short bilateral-penetration length $(<4 \mathrm{~nm})$ of topological edge states is detected, which tremendously reduces the distance limit in avoiding coupling between two parallel edge channels. Experimental evidences are also found in a multi-step area, where the edge states on different layers are sustained with a lateral separation of several nanometers. These results offer clear advantages for future developments of topological devices with 
robust performances and high channel density. Furthermore, our first-principles calculations reveal the contribution of surface hydrogenation to improve the quality of stanene films and the importance of the $\mathrm{Bi}$ substrate to endow the systems with robust nontrivial topologies. Apart from the topological property, we have also detected superconducting gaps of few-layer stanene using the $\mathrm{dI} / \mathrm{dV}$ spectra (STS). Fully superconducting gaps are present at 400 $\mathrm{mK}$ when the film thickness of stanene surpasses three layers. These results in few-layer stanene pave a shortcut to achieve a platform combining both topological edge states and superconductivity, two ingredients which further endow the systems with great potential to host the simplest 1D topological superconductors (TSCs)[20-22].

\section{Sample growth and STM characterization}

Stanene has a buckled honeycomb structure as shown in Fig. 1a. Based on our previous studies of experimentally growing stanene on Te-terminated $\mathrm{Bi}_{2} \mathrm{Te}_{3}$ substrate[10] and the theoretical prediction of achieving high-quality stanene film on Bi pre-covered $\mathrm{Bi}_{2} \mathrm{Te}_{3}$ surface[23], we have chosen $\mathrm{Bi}(111)$ as the substrate to grow stanene. As shown in Fig. 1b, the $\mathrm{Bi}(111)$ films were firstly grown on a silicon wafer with the film thickness of $\sim 10 \mathrm{~nm}$ to eliminate the epitaxial strain with silicon. Then an annealing process at $135^{\circ} \mathrm{C}$ has been applied to acquire large flat Bi (111) terraces (see Fig. 1c). The epitaxial growth of Sn was carried out right after the substrate was transferred to the preparation chamber from an environment of $4.2 \mathrm{~K}$ to keep a low deposition temperature, and the deposited Sn atoms evenly cover the $\mathrm{Bi}(111)$ surface (see Fig. 1c). After annealing at $40^{\circ} \mathrm{C}$, the $\mathrm{Sn}$ atoms form stanene films with good quality as shown in Fig. 1d (see Fig. S1 for details of sample growth). The profile along the black dotted line in Fig. 1d indicates the height of a single stanene layer of $\sim 0.38 \mathrm{~nm}$. The atomically resolved image consisting of the upper sublattice Sn atoms shows a hexagonal structure with a lattice constant of $\sim 0.455$ $\mathrm{nm}$, which is the same as that of the $\mathrm{Bi}(111)$ substrate. Multilayer stanene films have also been achieved here with good quality, except for the increased amount of Sn clusters of $1 \sim 2$ $\mathrm{nm}$ in height residing at the film edges (see Fig. 1e). Specifically, we have obtained 1-5 layer stanene films, and found that the atomic lattices show no visible difference on different layer films. We have further studied the stacking configuration of the stanene layers, and confirmed that it follows the structure of $\alpha$-Sn (see Fig. S2). It should also be emphasized that the top-most surface of the stanene films is saturated by hydrogen atoms based on previous experimental experiences[10, 12]. Our current experimental studies and systematic 
first-principles calculations further reveal that the surface passivation of the growth front by the residual hydrogen is essential in achieving layer-by-layer growth of the high-quality stanene films, with the hydrogen functioning as a surfactant[24, 25]. Details on the growth mechanism will be presented elsewhere.

\section{On the robust topological edge states}

The existence of an energy gap separating occupied and unoccupied states is essential for QSHIs[26]. Earlier theoretical studies have shown the potential existence of QSH states in freestanding stanene, including the effects of hydrogen passivation[4, 7]. Here we consider the physically realistic situation of stanene grown on the $\mathrm{Bi}(111)$ substrate both theoretically and experimentally. According to our present first-principles calculations (see Fig. S3 for the computational model), 1-5 layer stanene films on $\mathrm{Bi}(111)$ all have well-defined continuous gaps across the whole Brillouin zone (see Fig. S4), allowing to evaluate a topological invariant $Z_{2}$ for each system. The results are shown in the last row of Table I, indicating an extraordinarily robust nontrivial topology that is impervious to the layer thickness.

Given these strong theoretical results, we have experimentally investigated the potential existence of nontrivial topological edge states for different layer stanene films on $\mathrm{Bi}(111)$. Taking the 4-layer stanene as an example, we found that the inclusion of the spin-orbit coupling (SOC) removes the contact between the conduction and valcence bands (CBs and VBs) around the $\Gamma$ point and gives rise to a large continuous gap exceeding $200 \mathrm{meV}$ (see Fig. $2 \mathrm{a}$ and Fig. S5). Such a SOC-induced gap opening is accompanied by a topological phase transition and results in a nontrivial $Z_{2}$ number. In addition, the relatively low indirect overlapping of the CBs and VBs at different momenta characterizes the system to be a semimetal, as depicted by the calculated DOS in Fig. 2b. The dI/dV spectrum taken in the interior of the 4th-layer stanene film (hereafter referred to as the bulk) is also shown by the green curve. A shift of the Fermi level is expected because the theoretical model contains only 3 bilayer $\operatorname{Bi}(111)$ substrate, much thinner than the experimental thickness $(\sim 10 \mathrm{~nm})$. In our experiments, the thicker bismuth contributes to a higher electron doping level, resulting in an upward shift of the Fermi level relative to the calculated value. After adjusting the electron doping level, the calculated DOS matches well with the $\mathrm{dI} / \mathrm{dV}$ spectrum, especially within the energy window of -0.2 to $0.5 \mathrm{eV}$, overlapping the continuous gap. A remarkable feature in this range is the observed dip above the Fermi level, denoted as the bulk dip. Within this dip, the bulk states have the lowest density, thereby giving us a window to 
detect the potential evidences of the edge states. The spectra taken on stanene films with different layer thicknesses are compared in Fig. S4, all of which exhibit a similar dip feature.

The hallmark of the QSH systems is the topologically protected helical edge states, which are manifested by the enhanced intensity of local DOS (LDOS, obtained by the $\mathrm{dI} / \mathrm{dV}$ spectra) at the film edges in spatially-resolved STM studies [27-31]. For a semimetallic system, it is crucial to find an energy window in which the LDOS is dominated by topological edge states. Figure 2c shows a 4th-layer stanene island with a hexagonal shape. Two different zigzag edges are observed: the longer/shorter edge containing the Sn atoms of upper/lower sublattice (A/B edge in Fig. 1a, details to experimentally distinguish the A and B edges can be found in Fig. S2). We compared the dI/dV spectra taken in the bulk and at the two edges in Fig. 2d. The bulk spectrum shows the characteristic dip feature between 160 and $322 \mathrm{mV}$ as marked by the vertical gray dashed lines, and the minimum of bulk dip is located at $\sim 185 \mathrm{mV}$. At both edges, the intensities of the LDOS surpass that of the bulk spectrum essentially in the whole dip range. In particular, the spectrum of the A edge shows a distinct peak feature at $\sim 270 \mathrm{mV}$. The enhanced LDOS at both edges signifies the potential existence of the edge states, an important aspect to be more closely investigated below.

To visualize the energy distribution and spatial variation of the edge states, the spatially resolved relative differences of the LDOS [denoted by $\triangle$ LDOSs and defined as $\left(\right.$ LDOS-LDOS $\left._{b u l k}\right) /$ LDOS $_{b u l k}$, see Fig. S6 for details] across the A or B edge (along the black arrows in Fig. 2c) are shown in Fig. 2e. Clear evidences of the edge states can be observed as manifested by the localized red areas at both edges, but within different energy windows. The $\triangle$ LDOSs at both edges are projected on the sidewalls by the yellow and red curves, highlighting the dominance of the edge states within the energy windows of $165-285 \mathrm{meV}$ and $100-245 \mathrm{meV}$ at the $\mathrm{A}$ and $\mathrm{B}$ edges, respectively (denoted by the gray shadowed areas). To further display the spatial distrubtions of the electronic states within both windows over the whole island, a series of dI/dV mappings ranging from 100 to $322 \mathrm{meV}$ have been taken (Fig. 3a). According to the mappings, the LDOS at $100 \mathrm{meV}$ shows nearly an irregular distribution, except for a slight enhancement around the edges. At $185 \mathrm{meV}$, corresponding to the bulk dip minimum, the LDOS is highly pronounced at the edges, owing to the localized distribution of the edge states. We also notice the lack of interference pattern in the interior of the island, indicating the 1D character of the dominant electronic states 
at this energy. Further increasing the energy to $240 \mathrm{meV}$, the highlighted edge contour still exists but becomes disconnected, and some faint interference patterns emerge in the interior of the island as a signature of the surface states (namely, the bulk states defined earlier). At the peak energy of the A edge spectrum $(\sim 270 \mathrm{meV})$, only the A edges are still visible in the mapping, consistent with the $\mathrm{dI} / \mathrm{dV}$ spectra. These distinct states at the A edges contribute to the high energy part of the corresponding energy window, and may share similarities with the higher-order hinge states reported recently for bismuth films[30]. Besides, the enhanced interference patterns indicate the increased influences from the bulk states. Finally, at the peak energy of the bulk spectrum $(\sim 322 \mathrm{meV})$, the highlighted areas are inside the island and on the films with lower layer thickness, revealing that the bulk states dominate at this energy.

Based on the results presented above, we infer that the topological edge states exist within the energy windows of the A and B edges, especially highlighting itself around the bulk dip minimum. These experimental findings are also valid for stanene films with different layer thicknesses. Figure 3b shows the dI/dV mappings of the stanene films of 1-3 and 5 layers, taken at the respective bulk dip minima (residing within $180 \pm 20 \mathrm{meV}$ for different layer films, see Fig. S4). It is clear that all those mappings exhibit highlighted edge contours in the LDOS, demonstrating the existence of the edge states.

For the well-characterized 1D edge states near the bulk dip minimum, their topological nature needs to be further identified. To this end, we note that a topological edge state is typically accompanied by the linear crossings of electronic bands at some high-symmetry points in the momentum space, defining the Dirac points. In the present system, the calculated edge states of 4-layer stanene film show the existence of two Dirac points within each energy window of A and B edges mentioned above, labelled as D1-D4 in Fig. 2f. In particular, the corresponding topological edge states cover the bulk dip minimum in energy at both edges. Calculations of stanene films with different layer thicknesses also show the existence of topological edge states covering the bulk dip minimum (see Fig. S4). Based on the calculated results, we infer that the experimentally observed 1D edge states residing near the bulk dip minimum are topologically nontrivial.

Our STM studies and first-principles calculations indicate that the 1-5 layer stanene films on the $\mathrm{Bi}(111)$ substrate are all QSH systems. This observation is quite striking, because in typical situations, stacking of QSHI layers would alter the topological invariant and result in 
an odd-even oscillation of the $Z_{2}$ number[18]. This layer dependence of the topology is absent in our systems, at least up to 5 layers. As shown in Table I, the physical origin of the robust nontrivial $Z_{2}$ invariants against layer thickness is not due to the surface hydrogenation of the stanene films[7], but rather, is the consequence of their interfacial coupling with the $\mathrm{Bi}(111)$ substrate. Qualitatively, the $\mathrm{Bi}(111)$ substrate, with inherently strong $\mathrm{SOC}$, is expected to promote the nontrivial topology in the few-layer stanene via proximity effects[32, 33].

We have further experimentally studied the penetration depth of the topological edge states by integrating the spatially resolved $\triangle$ LDOSs over the repective energy windows for A and B edges (see details in Fig. S6). The results are displayed on the sidewalls by the blue curves in Fig. 2e, showing that the edge states have bilateral-penetration depths (including inner and outer sides) of less than $4 \mathrm{~nm}$ for the A edge and $5 \mathrm{~nm}$ for the B edge. In particular, towards the outer side of the A edge, the penetration depth is as short as $1 \mathrm{~nm}$. Such a short penetration depth outwards is understandable considering that the A edge is terminated by the Sn atoms in the upper sublattice, which have much weaker coupling with the lower layer stanene. In contrast, the B edge is composed of the Sn atoms in the lower sublattice, which are strongly coupled with the lower layer stanene, resulting in the nearly symmetric penetration depths outwards and inwards.

Considering that the A edges are generally longer and more regular than the B edges and have relatively shorter bilateral-penetration depths, it may enable the existence of parallel and densely packed multi-edge-channels, as conceptually depicted in Fig. 3f. Such a feasibility is partially conceived in a multi-step area shown in Fig. 3c. The profile of the gray dashed line is shown in Fig. 3d, by which we can recognize the height information as well as the distance between the neighboring steps. Here the steps have two different heights: single-layer and bilayer, indicated by the black and white lines, respectively, in Fig. 3c. The $\mathrm{dI} / \mathrm{dV}$ mapping shows the spatial distribution of the edge states in this area (see Fig. 3e). It is obvious that the edge states are regularly localized at the single-layer steps, even when the in-plane distance between them is less than $2 \mathrm{~nm}$. However, the edge states become irregular at the bilayer steps (see the area enclosed by the white dotted lines), where two edges are superposed without a well-defined in-plane distance. The irregular edge states are likely caused by the strong coupling and scattering between the edge states originating from superimposed edges. These results show that, on a principle level, few-layer stanene can be exploited to sustain dense multi-edge-channels using the A edge states of different layers 
when properly staggered with in-plane distances of several nanometers. The establishment of such channels further offers new opportunities for manipulation of topologically nontrivial states for potential applications in quantum devices.

\section{On the superconductivity}

Recently, superconductivity of few-layer stanene has been detected using transport measurements[15], and type-II Ising pairing has also been proposed to interpret the observed unusually large in-plane upper critical fields beyond the Pauli paramagnetic limit[16, 34]. Based on these earlier studies and the main results of the present work, we conjecture that simultaneous realization of superconductivity and topological edge states in stanene may be achieved in our systems, which in turn serves as an ideal platform for studying the interplay between the nontrivial topology and superconductivity, two central ingredients that can be further explored for realization of TSC[22, 35-37].

As shown in Fig. 4a, the superconductivity of stanene films grown on $\mathrm{Bi}(111)$ is experimentally confirmed by our STS measurements, where the superconducting gap shows significant layer dependence. Notably, the single-layer stanene can exhibit superconductivity once the separated small islands develop into a whole film. This difference with ref.[15] is likely caused by the higher electron doping levels from the metallic Bi(111) substrate. As the thickness of the stanene films increases, the superconducting gap becomes wider and deeper. Full gaps against thermal excitations at $400 \mathrm{mK}$ are obtained when the thickness reaches 3.5 layers or higher (see Fig. 4a). The gap values $(\Delta)$ extracted by half the difference of bias between the two coherence peaks are plotted in Fig. 4b, together with the zero bias conductance (ZBC) that quantizes the degree of the superconducting gap compared with a full gap. It can be seen that $\Delta$ increases rapidly with the thickness, saturating at $\sim 1.5$ layers; in contrast, ZBC decreases slower, saturating until $\sim 3.5$ layers.

Different from previous 2D material platforms combining nontrivial topological states and superconductivity which require the participation of the proximity effects associated with an additional s-wave superconductor[22, 38-40], or additional electrical gates[41, 42], both ingredients are inherently present in few-layer stanene. In particular, an inherent proximity effect would exist between the bulk and topological edge states of stanene itself as long as the edge states cross the Fermi level, resulting in the 1D TSC. This condition could be achieved after properly adjusting the doping level, e.g., by tuning the thickness of the substrate[15]. In addition, the intrinsically strong SOC may render stanene a promising 2D 
TSC under proper conditions[43]. The interplay between strong SOC, nontrivial topology, and superconductivity, together with the versatile tunability by surface functionalization or varying the substrate collectively make few-layer stanene a fertile ground for studying novel superconductivity and tunable topological properties for potential applications in quantum devices.

\section{Acknowledgements}

We thank the Ministry of Science and Technology of China (Grants No. 2019YFA0308600, 2020YFA0309000, 2016YFA0301003, No. 2016YFA0300403 No. 2017YFA0303500), NSFC (Grants No. 11521404, No. 11634009, No. 92065201, No. 11874256, No. 11874258, No. 12074247, No. 11790313, and No. 11861161003 No. 11634011, No. 11974323 , and No. 11722435), the Strategic Priority Research Program of Chinese Academy of Sciences (Grant No. XDB28000000 No. XDB30000000), the Science and Technology Commission of Shanghai Municipality (Grants No. 2019SHZDZX01, No. 19JC1412701, No. 20QA1405100) and Anhui Initiative in Quantum Information Technologies (Grant No. AHY170000) for partial support.

\section{Author contributions}

J. Jia and Z. Zhang co-designed the experimental project. C. Zhao conducted STM experiments and analyzed the data. J. Qin, H. Ma, B. Xia and B. Yang supported the STM experiments. L. Li calculated the band structure and analyzed the band topology. L. Zhang computationally optimized the structural models and related energetics. C. Zhao, L. Li, P. Cui, Z. Zhang and J. Jia wrote the paper. All authors discussed the results and commented on the manuscript.

[1] Hasan, M. Z. \& Kane, C. L. Colloquium: Topological insulators. Rev. Mod. Phys. 82, 3045-3067 (2010).

[2] Qi, X.-L. \& Zhang, S.-C. Topological insulators and superconductors. Rev. Mod. Phys. 83, 1057-1110 (2011).

[3] Xu, Y. et al. Large-Gap Quantum Spin Hall Insulators in Tin Films. Phys. Rev. Lett. 111, $136804(2013)$.

[4] Xu, Y., Tang, P. \& Zhang, S.-C. Large-gap quantum spin Hall states in decorated stanene 
grown on a substrate. Phys. Rev. B 92, 081112 (2015).

[5] Gou, J. et al. Strain-induced band engineering in monolayer stanene on Sb(111). Phys. Rev. Mater. 1, 054004 (2017).

[6] Wang, D., Chen, L., Wang, X., Cui, G. \& Zhang, P. The effect of substrate and external strain on electronic structures of stanene film. Phys. Chem. Chem. Phys. 17, 26979-26987 (2015).

[7] Chou, B.-H. et al. Hydrogenated ultra-thin tin films predicted as two-dimensional topological insulators. New J. Phys. 16, 115008 (2014).

[8] Yun, F., Cortie, D. L. \& Wang, X. Tuning the electronic structure in stanene/graphene bilayers using strain and gas adsorption. Phys. Chem. Chem. Phys. 19, 25574-25581 (2017).

[9] Liu, Y. et al. Realization of strained stanene by interface engineering. J. Phys. Chem. Lett. 10, 1558-1565 (2019).

[10] Zhu, F. et al. Epitaxial growth of two-dimensional stanene. Nat. Mater. 14, 1020-1025 (2015).

[11] Xu, C. et al. Gapped electronic structure of epitaxial stanene on $\operatorname{InSb}(111)$. Phys. Rev. B 97, $035122(2018)$.

[12] Zang, Y. et al. Realizing an epitaxial decorated stanene with an insulating bandgap. Advanced Functional Materials 28, 1802723 (2018).

[13] Deng, J. et al. Epitaxial growth of ultraflat stanene with topological band inversion. Nat. Mater. 17, 1081-1086 (2018).

[14] Zheng, X., Zhang, J.-F., Tong, B. \& Du, R.-R. Epitaxial growth and electronic properties of few-layer stanene on $\operatorname{InSb(111).~2D~Mater.~7,~} 011001$ (2019).

[15] Liao, M. et al. Superconductivity in few-layer stanene. Nat. Phys. 14, 344-348 (2018).

[16] Falson, J. et al. Type-ii ising pairing in few-layer stanene. Science 367, 1454-1457 (2020).

[17] Fu, L. \& Kane, C. L. Superconducting proximity effect and majorana fermions at the surface of a topological insulator. Phys. Rev. Lett. 100, 096407 (2008).

[18] Murakami, S. Quantum Spin Hall Effect and Enhanced Magnetic Response by Spin-Orbit Coupling. Phys. Rev. Lett. 97, 236805 (2006).

[19] Liu, C.-X. et al. Oscillatory crossover from two-dimensional to three-dimensional topological insulators. Phys. Rev. B 81, 041307 (2010).

[20] Nadjperge, S. et al. Observation of majorana fermions in ferromagnetic atomic chains on a superconductor. Science 346, 602-607 (2014). 
[21] Mourik, V. et al. Signatures of majorana fermions in hybrid superconductor-semiconductor nanowire devices. Science 336, 1003-1007 (2012).

[22] Jäck, B. et al. Observation of a majorana zero mode in a topologically protected edge channel. Science 364, 1255-1259 (2019).

[23] Zhang, L. et al. Kinetic pathways towards mass production of single crystalline stanene on topological insulator substrates. Nanoscale 10, 18988-18994 (2018).

[24] Copel, M., Reuter, M. C., Kaxiras, E. \& Tromp, R. M. Surfactants in epitaxial growth. Phys. Rev. Lett. 63, 632-635 (1989).

[25] Zhang, Z. \& Lagally, M. G. Atomistic processes in the early stages of thin-film growth. Science 276, 377-383 (1997).

[26] Bernevig, B. A., Hughes, T. L. \& Zhang, S.-C. Quantum Spin Hall Effect and Topological Phase Transition in HgTe Quantum Wells. Science 314, 1757-1761 (2006).

[27] Drozdov, I. K. et al. One-dimensional topological edge states of bismuth bilayers. Nat. Phys. 10, 664-669 (2014).

[28] Tang, S. et al. Quantum spin Hall state in monolayer 1T'-WTe 2 . Nat. Phys. 13, 683-687 (2017).

[29] Collins, J. L. et al. Electric-field-tuned topological phase transition in ultrathin $\mathrm{Na}_{3} \mathrm{Bi}$. Nature 564, 390-394 (2018).

[30] Schindler, F. et al. Higher-order topology in bismuth. Nat. Phys. 14, 918-924 (2018).

[31] Zhao, C. et al. Strain Tunable Semimetal-Topological-Insulator Transition in Monolayer 1T'$\mathrm{WTe}_{2}$. Phys. Rev. Lett. 125, 046801 (2020).

[32] Liu, Z. et al. Stable Nontrivial $Z_{2}$ Topology in Ultrathin Bi(111) Films: A First-Principles Study. Phys. Rev. Lett. 107, 136805 (2011).

[33] Wu, G. et al. Tuning the vertical location of helical surface states in topological insulator heterostructures via dual-proximity effects. Sci. Rep. 3, 1233 (2013).

[34] Clogston, A. M. Upper limit for the critical field in hard superconductors. Phys. Rev. Lett. 9, 266 (1962).

[35] Sun, H.-H. et al. Majorana zero mode detected with spin selective andreev reflection in the vortex of a topological superconductor. Phys. Rev. Lett. 116, 257003 (2016).

[36] Wang, D. et al. Evidence for majorana bound states in an iron-based superconductor. Science 362, 333-335 (2018). 
[37] Zhang, P. et al. Observation of topological superconductivity on the surface of an iron-based superconductor. Science 360, 182-186 (2018).

[38] Sun, H.-H. et al. Coexistence of topological edge state and superconductivity in bismuth ultrathin film. Nano Lett. 17, 3035-3039 (2017).

[39] Hart, S. et al. Induced superconductivity in the quantum spin Hall edge. Nat. Phys. 10, 638-643 (2014).

[40] Lüpke, F. et al. Proximity-induced superconducting gap in the quantum spin Hall edge state of monolayer $\mathrm{WTe}_{2}$. Nat. Phys. 16, 526-530 (2020).

[41] Sajadi, E. et al. Gate-induced superconductivity in a monolayer topological insulator. Science 362, 922-925 (2018).

[42] Fatemi, V. et al. Electrically tunable low-density superconductivity in a monolayer topological insulator. Science 362, 926-929 (2018).

[43] Wang, J., Xu, Y. \& Zhang, S.-C. Two-dimensional time-reversal-invariant topological superconductivity in a doped quantum spin-hall insulator. Phys. Rev. B 90, 054503 (2014). 
FIGURES

331 

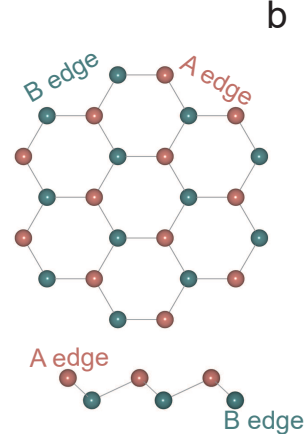

- Upper Sn

- Lower Sn

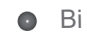

- $\mathrm{H}$

d
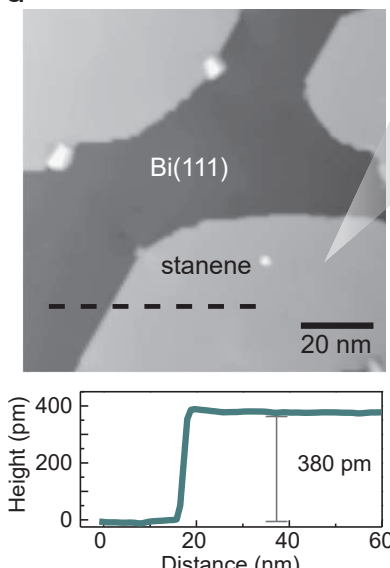
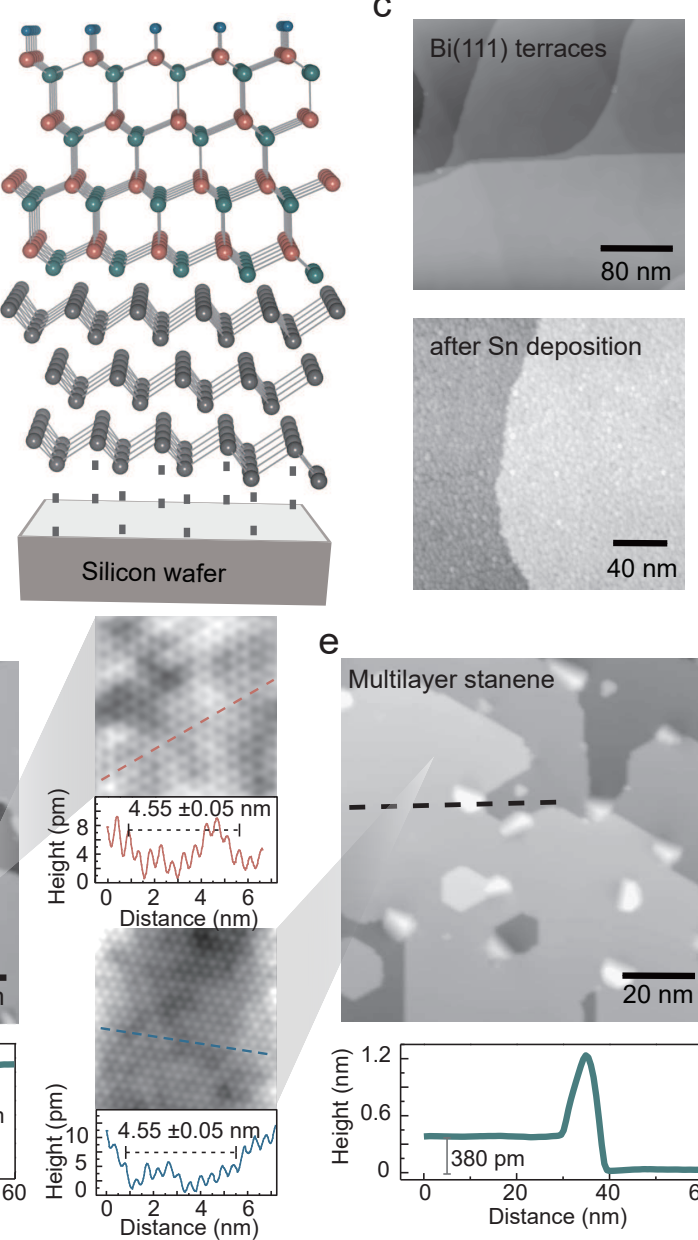

e
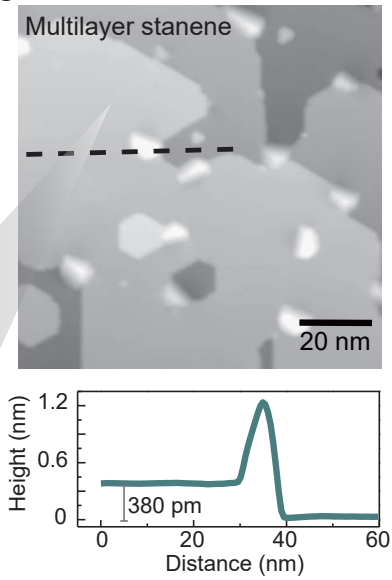

FIG. 1. Stanene films grown on the Bi(111) substrate. a, Schematics of a freestanding monolayer stanene containing two sublattices of Sn atoms: top view (upper panel) and side view (lower panel). b, Schematic of the sample structure of 4-layer stanene/Bi(111)/Si, in which only 3 bilayer $\mathrm{Bi}(111)$ films are explicitly displayed. The top surface of stanene is passivated by hydrogen atoms. c, Topography of the multilayered Bi(111) film before (upper panel) and after (lower panel) depositing Sn at low temperature. d, Topography of the 1st-layer stanene films formed on $\operatorname{Bi}(111)$ after annealing at $\sim 40{ }^{\circ} \mathrm{C}$, with a cut line depicting the height profile shown at the bottom. The atomically resolved image shown on the upper middle displays the surface lattice of the 1st-layer stanene. e, Topography of multilayer stanene ( 2.5 layers $)$ on $\operatorname{Bi}(111)$, with a cut line depicting the height profile shown at the bottom. Half layer means the half deposition time of Sn atoms compared with that of one layer. The atomically resolved image of the 3rd-layer stanene shown on the lower middle displays its surface lattice constant. 


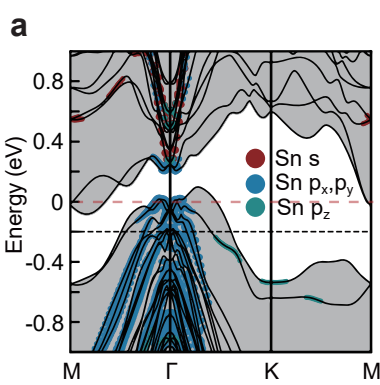

b

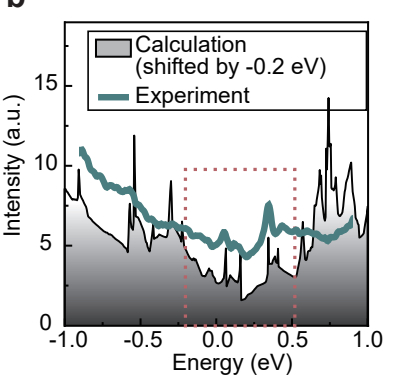

c
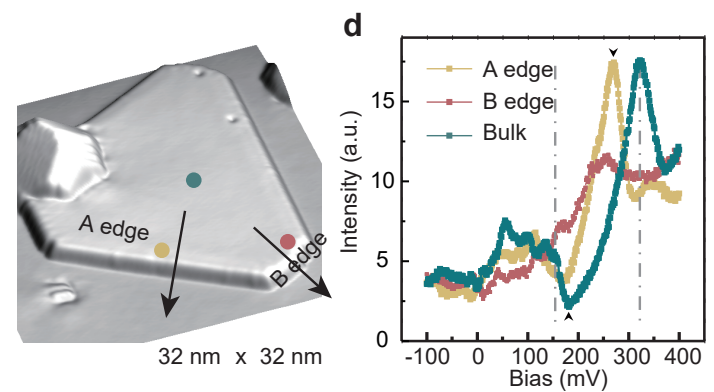
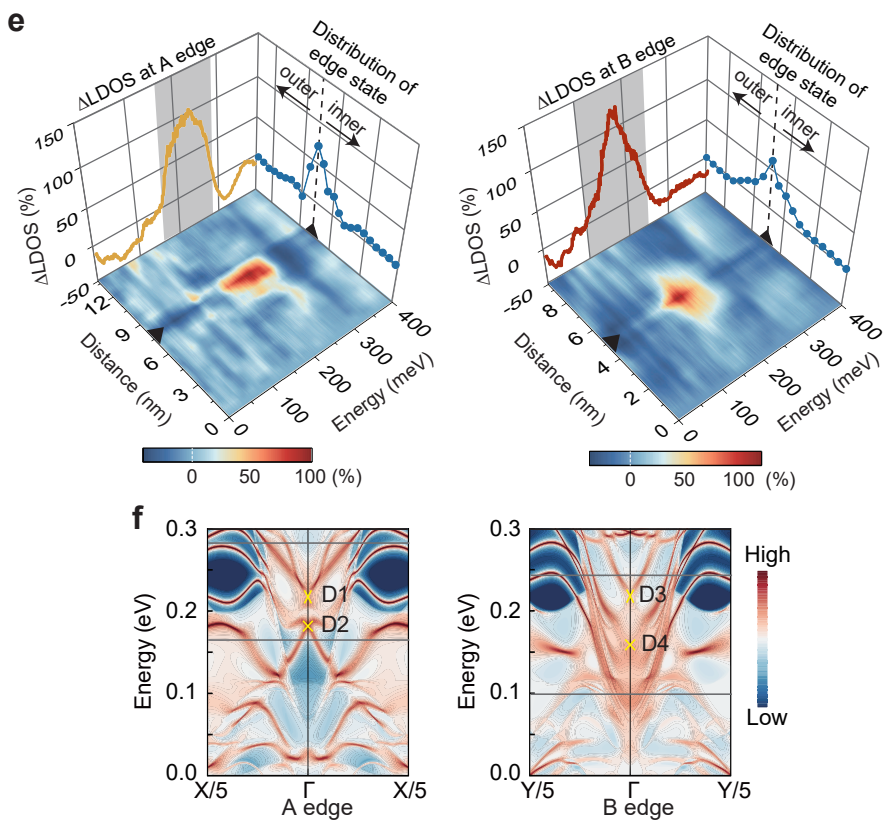
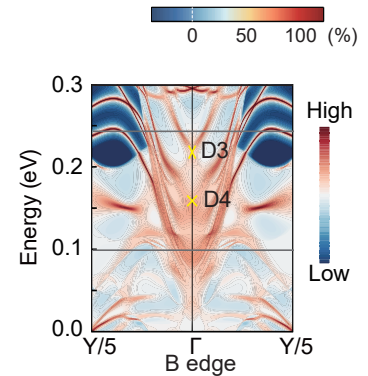

FIG. 2. Analysis of electronic structures and edge states of multilayer stanene on Bi(111). a, Calculated band structure of 4-layer stanene film on $\mathrm{Bi}(111)$ with contributions from different Sn orbitals highlighted by spots in different colors. An upward shift of $0.2 \mathrm{eV}$ is applied to the calculated Fermi level (black dotted line) owing to the different electron doping level between experiments and calculations. b, Comparison between calculated DOS and experimentally detected $\mathrm{dI} / \mathrm{dV}$ spectra on the 4-layer stanene film on $\mathrm{Bi}(111)$. The dotted lines highlight the well-matched low energy range around the Fermi level. c, Topography of a 4th-layer stanene island with a hexagonal shape. $\mathbf{d}, \mathrm{dI} / \mathrm{dV}$ spectra taken in the interior and at the $\mathrm{A}$ or $\mathrm{B}$ edge of the same stanene island shown in c, as marked by the different colored spots. e, Three-dimensional plots of the $\triangle$ LDOSs as a function of energy and spatial distance along the black arrows in $\mathbf{c}$, for $\mathrm{A}$ (left) and B (right) edges. The $\triangle$ LDOSs at respective edges (marked out by the black triangles) are projected on the sidewalls using the same color codes with $\mathbf{d}$ with the gray shadowed areas showing the energy windows dominated by the edge states $(\Delta \operatorname{LDOS}>0)$. The spatial variations of the edge states integrating over respective energy windows are also projected by the blue curves. f, Calculated topological edge states at the A and B edges, with the Dirac points D1-D4 marked by the yellow crosses. The gray shadowed energy windows in e are indicated by the horizontal gray lines. 


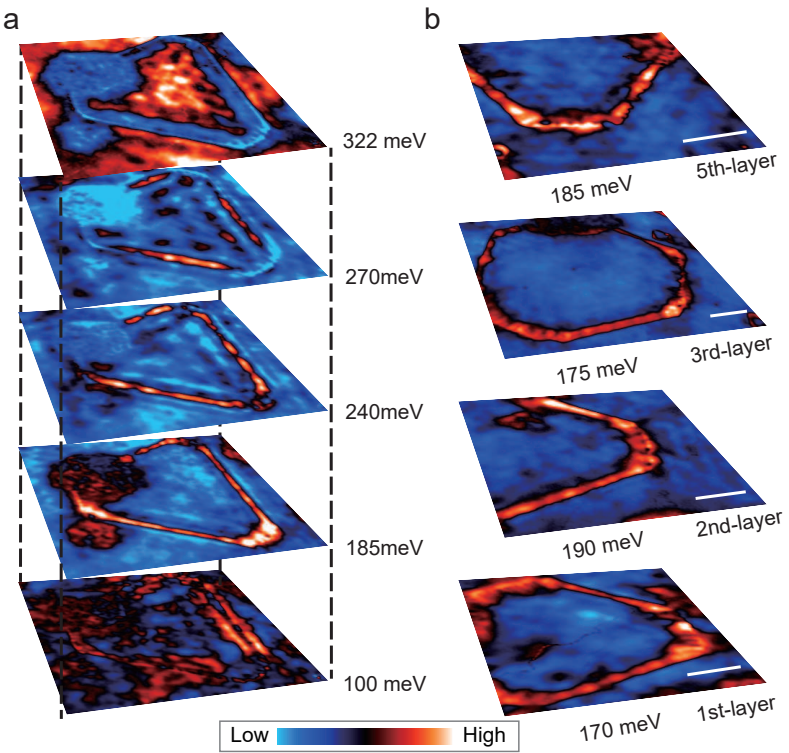

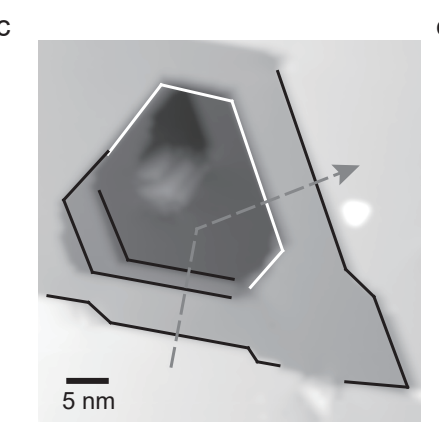

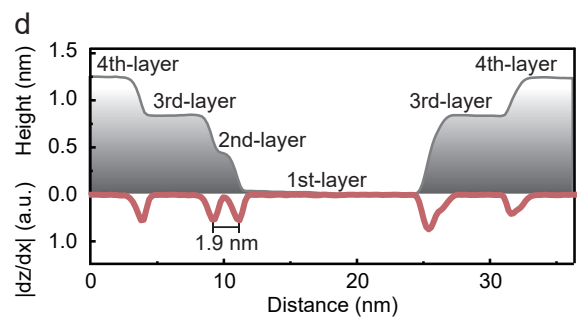

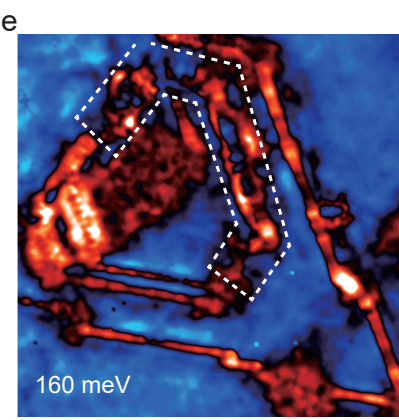

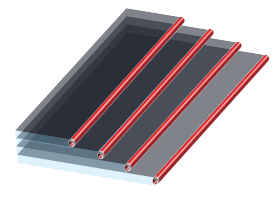

FIG. 3. Spatial distributions of topological edge states. a, dI/dV mappings of the stanene island shown in Fig. 2c at different energies. b, dI/dV mappings of stanene islands on different layers taken at the energy of the respective bulk dip minimum. Scale bars: $5 \mathrm{~nm}$. c, Topography of a multi-step area. Steps with a single-layer (bilayer) height are depicted by the black (white) lines. d, Profile of the gray dashed lines in $\mathbf{c}$, with the absolute value of the slope of the height profile defined by $|\mathrm{dz} / \mathrm{dx}|$ shown at the bottom measuring the distance between the neighboring steps. e, $\mathrm{dI} / \mathrm{dV}$ mapping of the multi-step area at the energy near the bulk dip minimum. f, Schematic graph of multi-edge-channels (red) along the properly staggered A edges. 

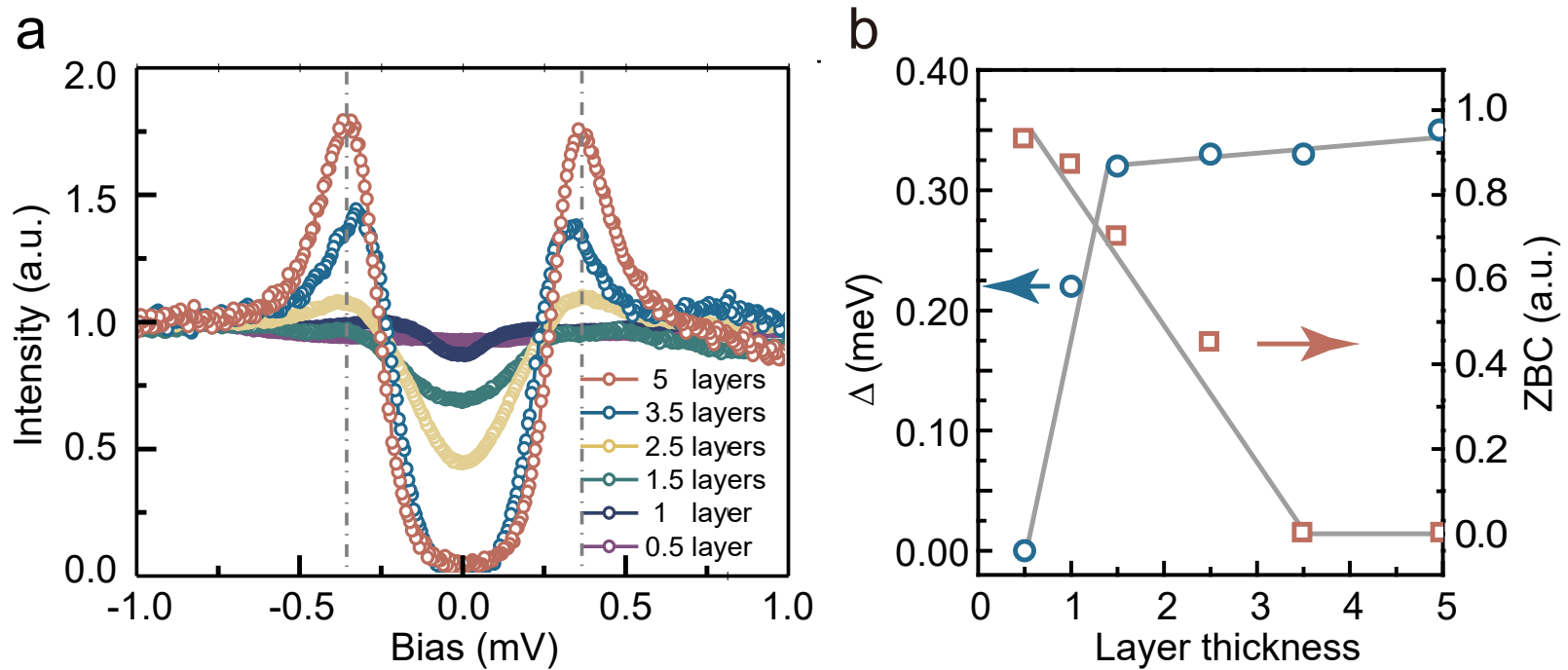

FIG. 4. Superconductivity of stanene films. a, Layer-dependent superconducting gap of stanene films at $400 \mathrm{mK}$, normalized by the intensity of the normal states at $-1.0 \mathrm{mV}$. b, Variations of the SC gap value $(\Delta)$ and the ZBC with the layer thickness. 
TABLES

377 
TABLE I. $Z_{2}$ invariants of different layer stanene films under various conditions.

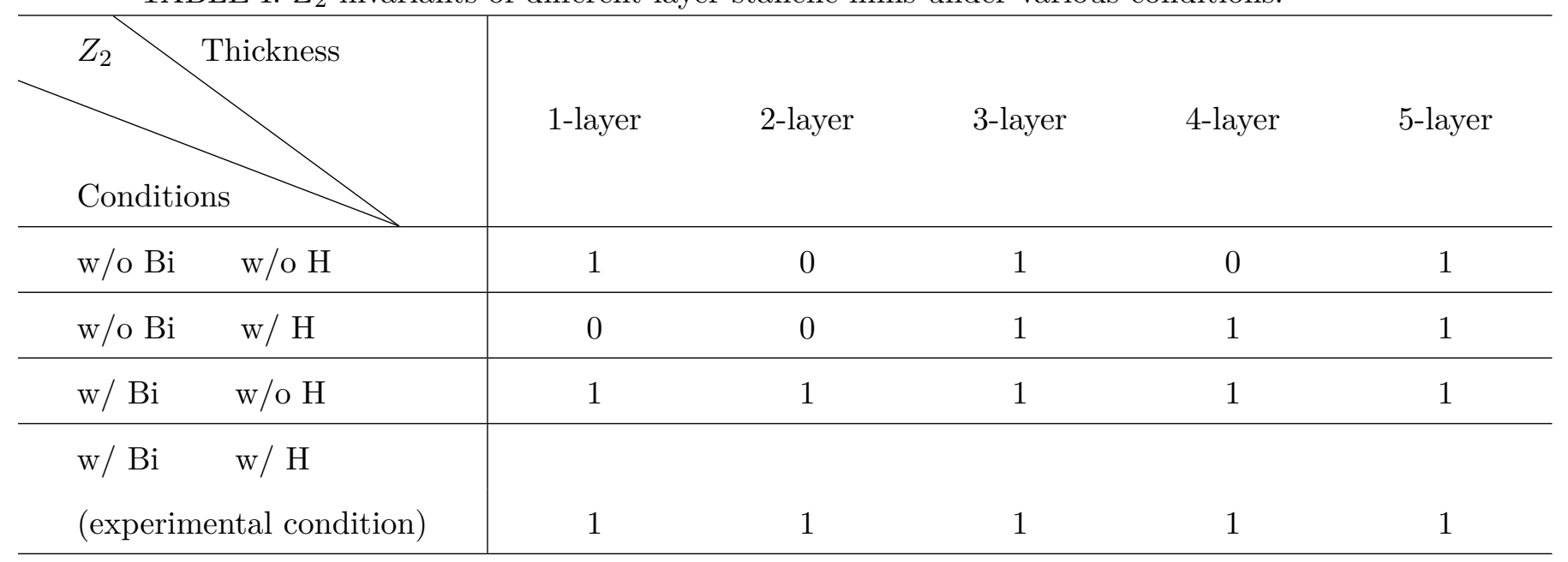




\section{Figures}

a

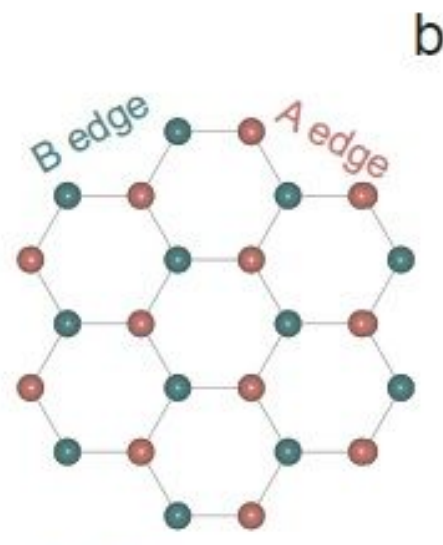

A edge $\bullet$ B edge

- Upper Sn - Bi

- Lower Sn • H

b
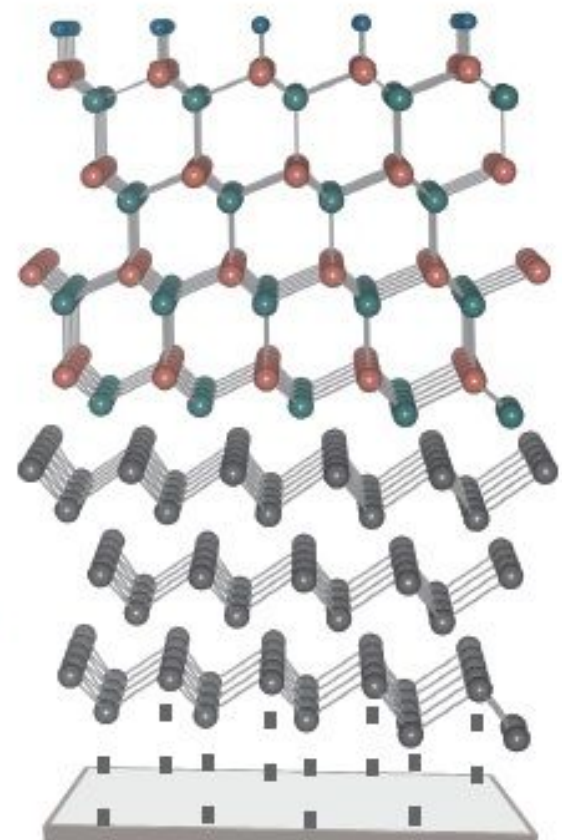

Silicon wafer
C
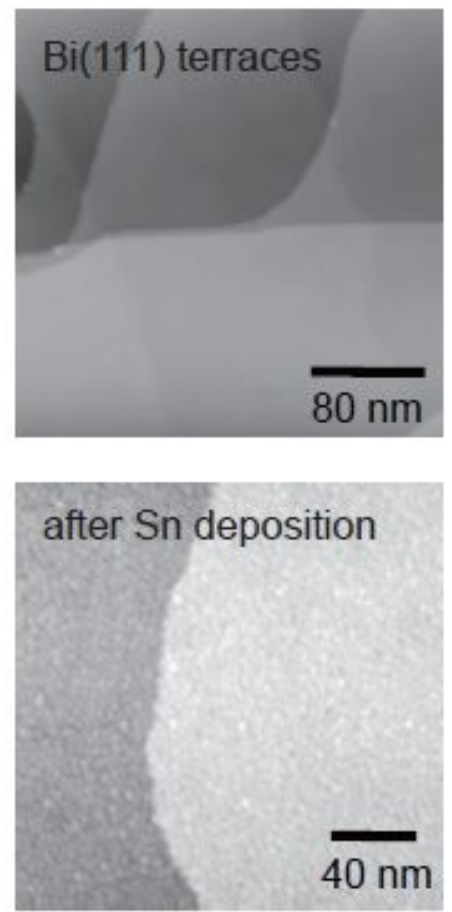

d
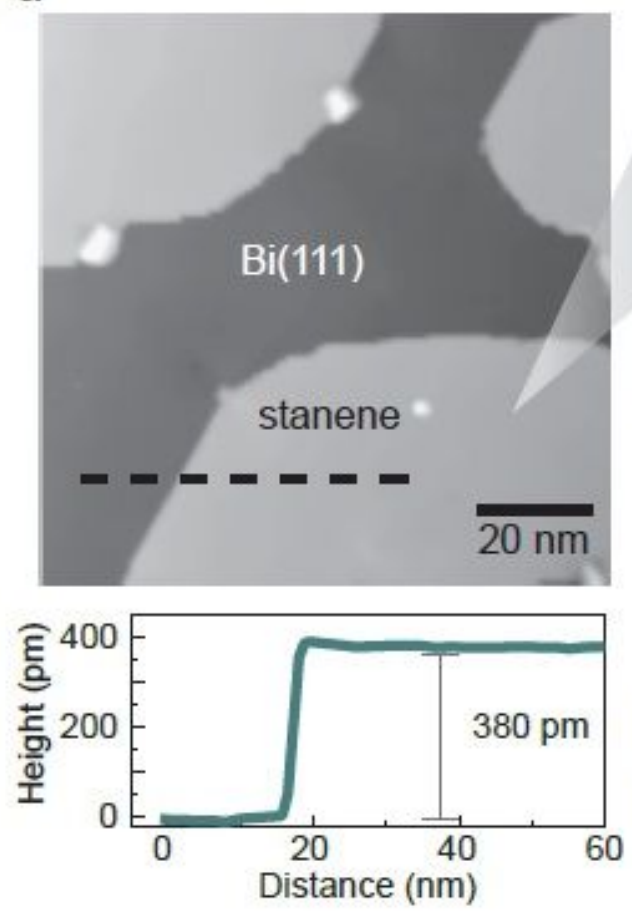

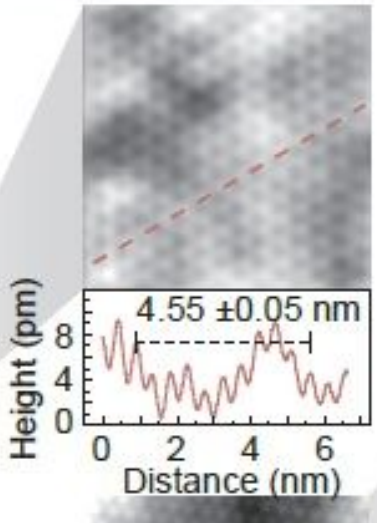

e
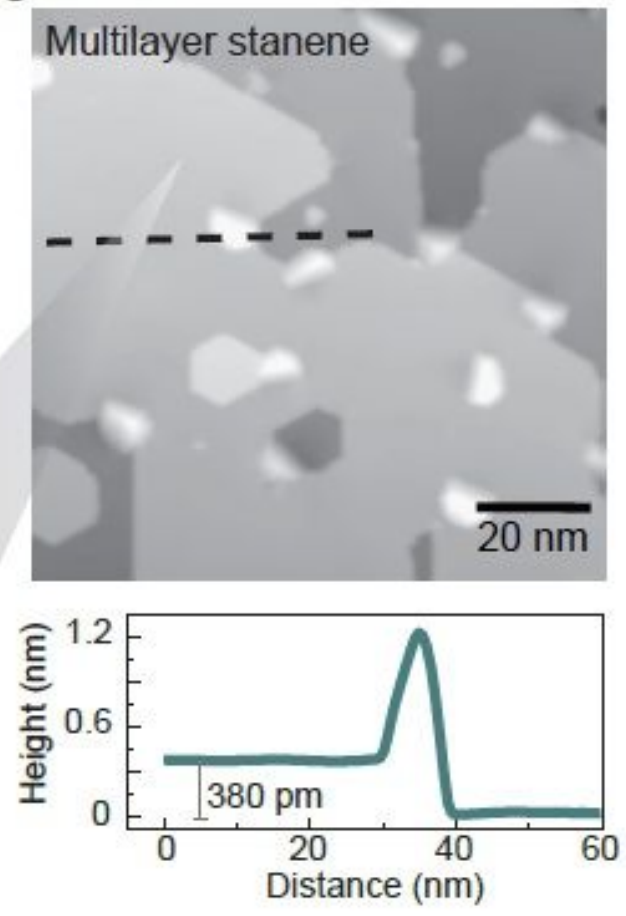

\section{Figure 1}

Stanene films grown on the $\mathrm{Bi}(111)$ substrate. a, Schematics of a freestanding monolayer stanene containing two sublattices of Sn atoms: top view (upper panel) and side view (lower panel). b, Schematic of the sample structure of 4-layer stanene/Bi(111)/Si, in which only 3 bilayer $\mathrm{Bi}(111)$ films are explicitly 
displayed. The top surface of stanene is passivated by hydrogen atoms. c, Topography of the multilayered $\mathrm{Bi}(111)$ film before (upper panel) and after (lower panel) depositing $\mathrm{Sn}$ at low temperature. $\mathrm{d}$, Topography of the 1 st-layer stanene films formed on $\mathrm{Bi}(111)$ after annealing at $\varangle 40 \otimes \mathrm{C}$, with a cut line depicting the height profile shown at the bottom. The atomically resolved image shown on the upper middle displays the surface lattice of the 1st-layer stanene. e, Topography of multilayer stanene $(\mathbb{Z} 2.5$ layers) on $\mathrm{Bi}(111)$, with a cut line depicting the height profile shown at the bottom. Half layer means the half deposition time of $\mathrm{Sn}$ atoms compared with that of one layer. The atomically resolved image of the 3rd-layer stanene shown on the lower middle displays its surface lattice constant.
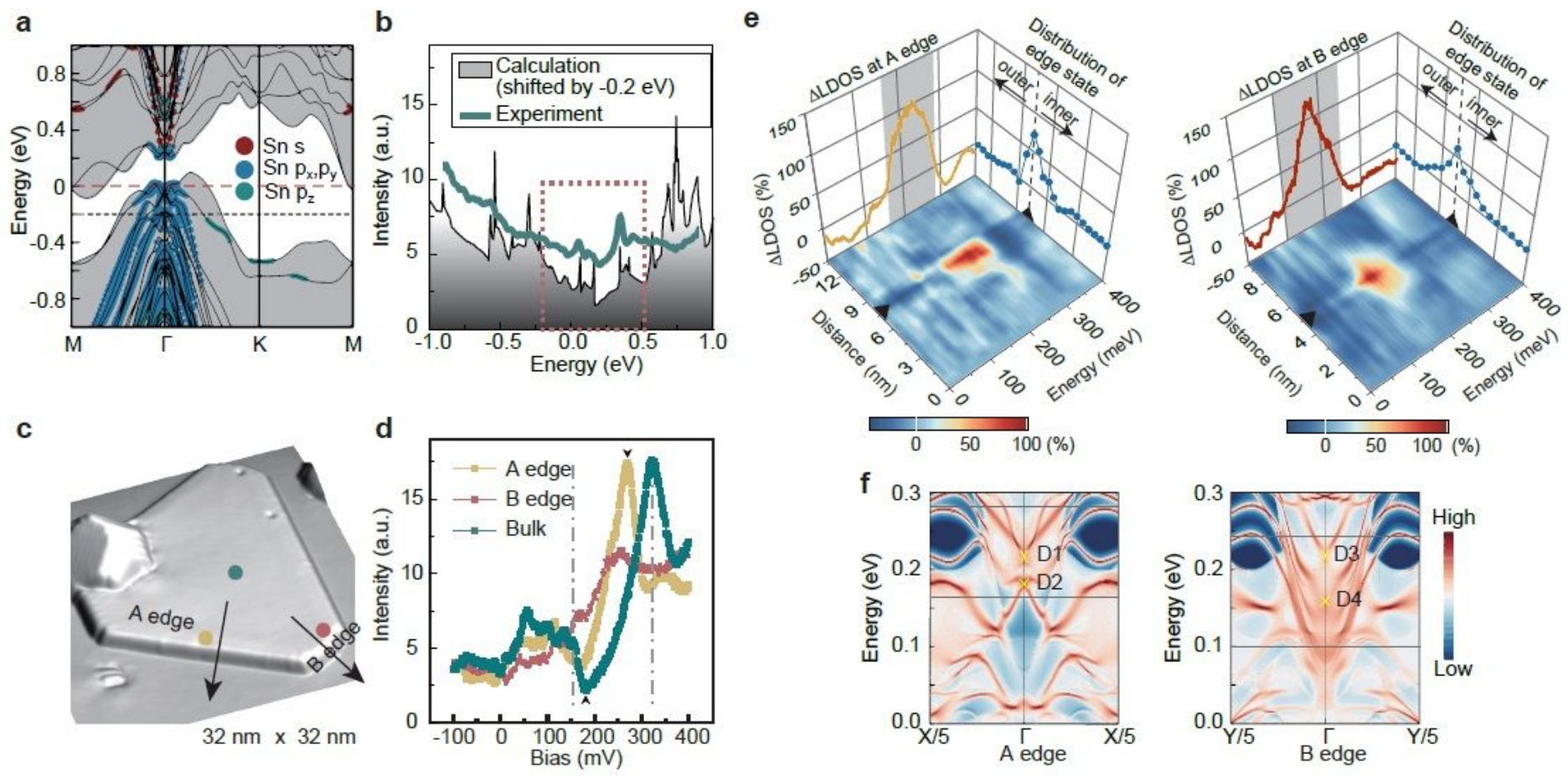

\section{Figure 2}

Analysis of electronic structures and edge states of multilayer stanene on $\mathrm{Bi}(111)$. a, Calculated band structure of 4-layer stanene film on $\mathrm{Bi}(111)$ with contributions from different $\mathrm{Sn}$ orbitals highlighted by spots in different colors. An upward shift of $0.2 \mathrm{eV}$ is applied to the calculated Fermi level (black dotted line) owing to the different electron doping level between experiments and calculations. b, Comparison between calculated DOS and experimentally detected dl/dV spectra on the 4-layer stanene film on $\mathrm{Bi}(111)$. The dotted lines highlight the well-matched low energy range around the Fermi level. $\mathrm{c}$, Topography of a 4th-layer stanene island with a hexagonal shape. $\mathrm{d}, \mathrm{dl} / \mathrm{dV}$ spectra taken in the interior and at the A or B edge of the same stanene island shown in c, as marked by the different colored spots. $\mathrm{e}$, Three-dimensional plots of the $\triangle \mathrm{LDOSs}$ as a function of energy and spatial distance along the black arrows in c, for A (left) and B (right) edges. The $\triangle$ LDOSs at respective edges (marked out by the black triangles) are projected on the sidewalls using the same color codes with $\mathrm{d}$ with the gray shadowed areas showing the energy windows dominated by the edge states $(\Delta \mathrm{LDOS}>0)$. The spatial variations of the edge states integrating over respective energy windows are also projected by the blue curves. 

yellow crosses. The gray shadowed energy windows in e are indicated by the horizontal gray lines.

a

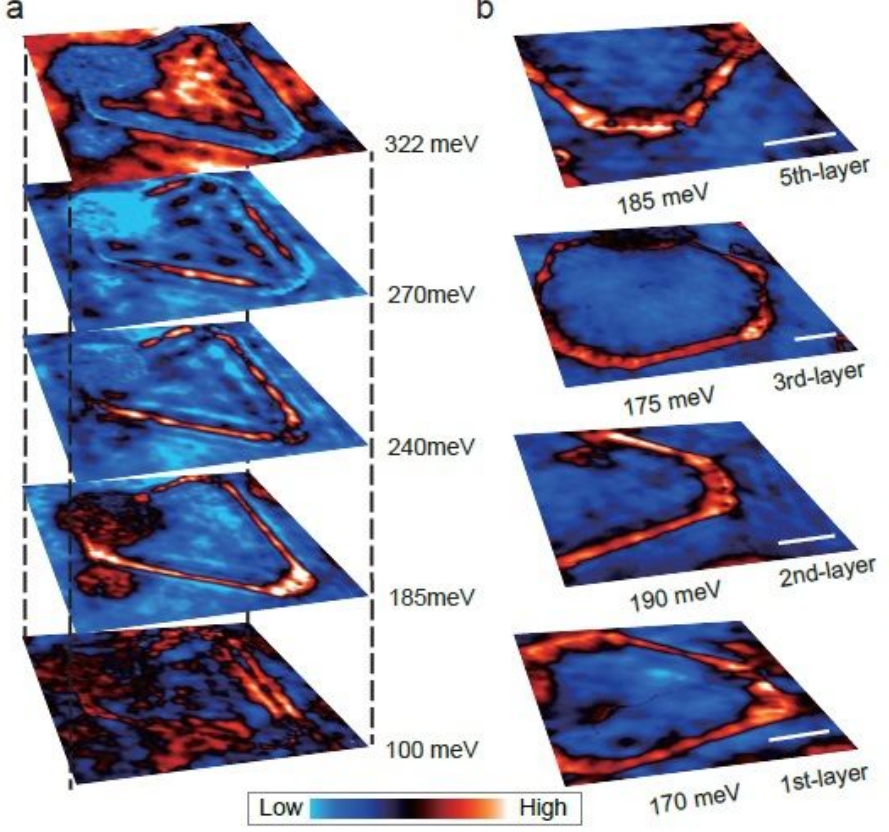

C

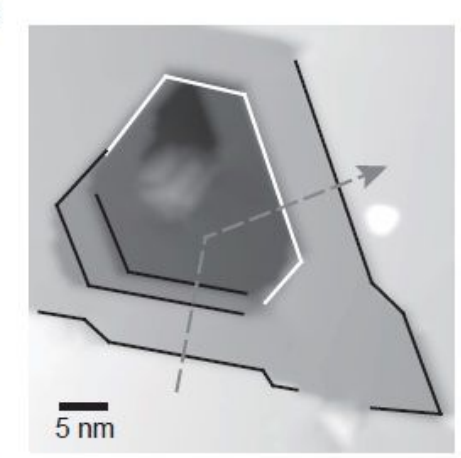

d

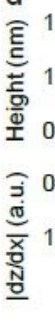

e

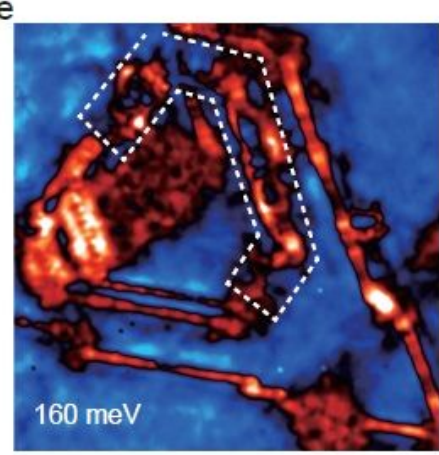

$f$

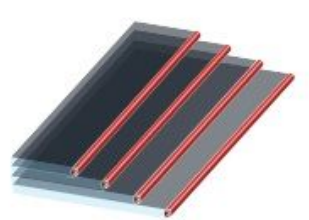

\section{Figure 3}

Spatial distributions of topological edge states. a, dl/dV mappings of the stanene island shown in Fig. 2c at different energies. $b, \mathrm{dl} / \mathrm{dV}$ mappings of stanene islands on different layers taken at the energy of the respective bulk dip minimum. Scale bars: $5 \mathrm{~nm}$. c, Topography of a multi-step area. Steps with a singlelayer (bilayer) height are depicted by the black (white) lines. d, Profile of the gray dashed lines in c, with the absolute value of the slope of the height profile defined by $|d z / d x|$ shown at the bottom measuring the distance between the neighboring steps. e, $\mathrm{dl} / \mathrm{dV}$ mapping of the multi-step area the energy near the bulk dip minimum. $\mathrm{f}$, Schematic graph of multi-edge-channels (red) along the properly staggered $A$ edges. 

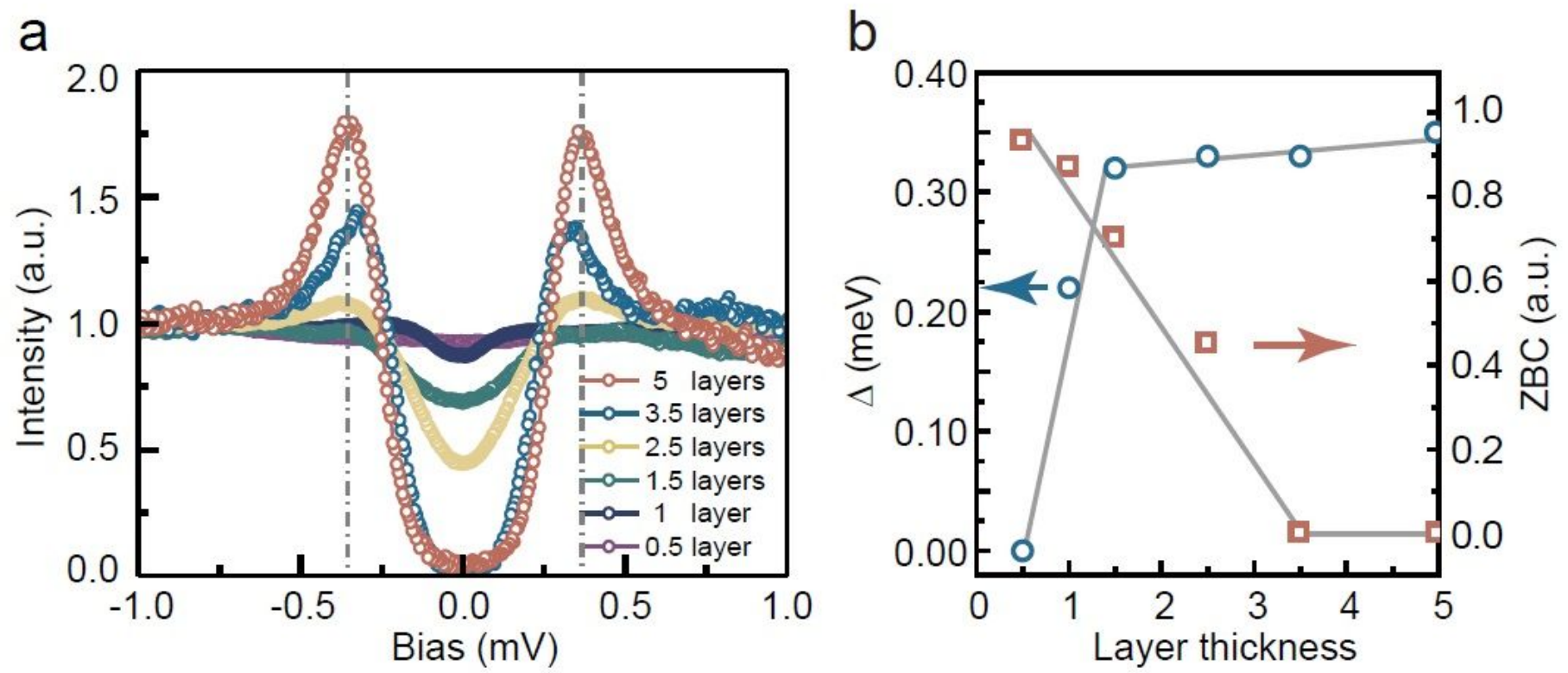

Figure 4

Superconductivity of stanene films. a, Layer-dependent superconducting gap of stanene films at $400 \mathrm{mK}$, normalized by the intensity of the normal states at $-1.0 \mathrm{mV}$. b, Variations of the SC gap value $(\Delta)$ and the ZBC with the layer thickness.

\section{Supplementary Files}

This is a list of supplementary files associated with this preprint. Click to download.

- Supplementary.pdf 